\title{
Resistência Antimicrobiana: Aspectos Clínicos e Econômicos nos Custos da Hospitalização
}

\section{Antimicrobial Resistance: Clinical and Economic Aspects of Hospitalization Costs}

\author{
Nayane Laine Paglione ${ }^{1}$ \\ Renne Rodrigues ${ }^{2}$ \\ Marcia Regina Eches Perugini ${ }^{3}$ \\ Maria do Carmo Fernandez Lourenco Haddad ${ }^{4}$ \\ Gilselena Kerbauy ${ }^{5}$
}

\section{RESUMO}

Objetivo: Avaliar os aspectos clínicos e econômicos da resistência antimicrobiana em hemocultura nos custos diretos de pacientes hospitalizados. Metodologia: Estudo retrospectivo, transversal, realizado em hospital terciário, no Sul do Brasil. A amostra foi composta por pacientes com hemocultura positiva, idade $\geq 14$ anos, hospitalizados por período $\geq 48$ horas entre janeiro e dezembro de 2017. Foram considerados microrganismos resistentes bactérias resistentes à oxacilina, vancomicina, carbapenêmicos, cefalosporinas, fluorquinolonas e aminoglicosídeos. Os custos diretos da hospitalização foram cedidos pelo setor financeiro da instituição. Os dados foram analisados pelo programa SPSS ${ }^{\circledR}$ versão 20. Resultados: A amostra do estudo foi composta por 261 pacientes, destes $62,45 \%$ apresentavam microrganismo resistente na hemocultura. A resistência antimicrobiana foi mais frequente em pacientes internados em unidade de terapia intensiva $(p=0,0017)$ e que permaneceram neste setor por mais de 15 dias $(p<0,001)$. A hemocultura por microrganismo resistente aumentou em duas vezes as chances de mortalidade quando comparadas aos pacientes com hemocultura por microrganismo sensível $(p=0,0016)$. Os custos diretos atribuídos à hospitalização foram maiores em pacientes com hemocultura por microrganismo resistente, em comparação aos com microrganismo sensível $(p<0,001)$. Conclusão: A resistência antimicrobiana em hemoculturas foi associada à hospitalização prolongada em unidade de terapia intensiva, mortalidade e aumento dos custos diretos da hospitalização.

\section{DESCRITORES}

Farmacorresistência Bacteriana Múltipla. Custos de Cuidados de Saúde. Custos de Medicamentos. Infecção Hospitalar.

\begin{abstract}
Objective: To evaluate the clinical and economic aspects of antimicrobial resistance in blood culture in the direct costs of hospitalized patients. Methodology: Retrospective, cross-sectional study carried out at a tertiary hospital in southern Brazil. The sample consisted of patients with positive blood culture, aged $\geq 14$ years, hospitalized for a period of $\geq 48$ hours between January and December 2017. Microorganisms resistant to oxacillin, vancomycin, carbapenems, cephalosporins, fluoroquinolones and aminoglycosides were considered resistant. The direct costs of hospitalization were provided by the institution's financial department. Data were analyzed using SPSS $₫$ version 20 software. Results: The study sample consisted of 261 patients, of these $62.45 \%$ presented resistant microorganism in the blood culture. Antimicrobial resistance was more frequent in intensive care unit patients $(p=0.0017)$ with hospitalization in this unit for more than 15 days $(p<0.001)$. Resistant microorganism blood culture increased the odds of mortality twice as compared to patients with sensitive microorganism blood culture $(p=0.0016)$. The direct costs attributed to hospitalization were higher in patients with resistant microorganism blood culture compared to sensitive microorganism $(p<0.001)$. Conclusion: Antimicrobial resistance in blood cultures was associated with prolonged intensive care unit hospitalization, mortality, and increased direct hospitalization costs.
\end{abstract}

\section{DESCRIPTORS}

Multiple Bacterial Drug Resistance. Health Care Costs. Drug Costs. Cross Infection.

${ }^{1}$ Enfermeira, Mestre em Enfermagem, Universidade Estadual de Londrina, Londrina, Paraná, Brasil.

${ }^{2}$ Farmacêutico, Doutor em Saúde Coletiva, Docente do Departamento de Saúde Coletiva, Universidade Estadual de Londrina, Londrina, Paraná, Brasil.

${ }^{3}$ Bióloga, Doutora em Microbiologia, Docente do Departamento de Análises Clínicas e Toxicologia, Universidade Estadual de Londrina, Londrina, Paraná, Brasil.

${ }^{4}$ Enfermeira, Doutora em Enfermagem, Docente do Departamento de Enfermagem, Universidade Estadual de Londrina, Londrina, Paraná, Brasil.

${ }^{5}$ Enfermeira, Doutora em Microbiologia, Docente do Departamento de Enfermagem, Universidade Estadual de Londrina, Londrina, Paraná, Brasil. 
$\mathrm{O}$ s antimicrobianos salvaram milhões de vidas e transformaram os cuidados em saúde, entretanto estão se tornando menos eficazes devido à resistência antimicrobiana e a escassez no desenvolvimento de novos fármacos ${ }^{1}$.

Este fato tornou a resistência antimicrobiana uma das prioridades da Organização Mundial da Saúde, considerando que os microrganismos multirresistentes aos antimicrobianos (MOMR) ameaçam séculos de avanços na área da saúde e colocam em risco a saúde da humanidade ${ }^{2}$.

A resistência antimicrobiana é responsável por pelo menos 700.000 mortes ao ano em todo o mundo. Estima-se que este número cresça de forma alarmante até 2050, quando aproximadamente 2,4 milhões de pessoas poderão morrer em virtude de infecções resistentes a estes fármacos ${ }^{2}$.

Além de representar risco à vida humana, a resistência antimicrobiana contribui para onerar o orçamento dos sistemas de saúde, sejam eles públicos ou privados ${ }^{3}$.

O tratamento de infecções desencadeadas por microrganismos resistentes a antimicrobianos requer terapias prolongadas e mais dispendiosas, além de aumentar o período de hospitalização4.

Estimativas indicam que a resistência aos antimicrobianos provocará danos à economia tão catastróficos que poderá levar 24 milhões de pessoas à pobreza extrema, devido ao aumento com os gastos em saúde ${ }^{2}$.

Considerando a relevância do tema e a escassez de dados brasileiros referentes à temática, este estudo objetivou avaliar os aspectos clínicos e econômicos da resistência antimicrobiana nos custos diretos de pacientes hospitalizados.

\section{METODOLOGIA}

Estudo retrospectivo, transversal, com abordagem quantitativa, que se propôs a comparar os indicadores clínicos e os custos diretos da hospitalização entre pacientes infectados por microrganismos resistentes e sensíveis aos antimicrobianos.

O local do estudo foi um hospital terciário, de alta complexidade, localizado na $\mathrm{Re}$ gião Sul do Brasil, que realiza atendimento a pacientes clínicos e cirúrgicos. É composto por 191 leitos, sendo 45 leitos de terapia intensiva. Aproximadamente, $80 \%$ dos leitos totais são destinados ao sistema único de saúde (SUS).

Foram incluídos todos os pacientes com idade igual ou superior a 14 anos, hospitalizados no período de janeiro a dezembro de 2017 por tempo superior a 48 horas, em setores clínicos, cirúrgicos ou de terapia intensiva, com diagnóstico microbiológico positivo para bactérias em hemoculturas. Foram excluídos pacientes que apresentavam hemocultura com crescimento para Estafilococos coagulase negativa, pois os mesmos podem ser considerados contaminantes da amostra por se tratar de um microrganismo tipicamente comensal de pele. Na maioria das vezes este resultado é devido a falhas na técnica asséptica de coleta das hemoculturas ${ }^{5}$.

As variáveis clínicas estudadas foram: sexo, idade, setor e período de hospitalização, permanência em unidade de terapia intensiva (UTI), classificação da internação (clínica ou cirúrgica) e desfecho clínico (óbito e alta). As variáveis microbiológicas consideraram a espécie bacteriana e o perfil de sensibilidade aos antimicrobianos.

A técnica de coleta das hemoculturas 
na instituição é padronizada e foi realizada por auxiliares e técnicos em laboratório. As amostras de sangue coletadas foram inoculadas em frascos contendo meio de cultura (BD BACTEC $^{\text {TM }}$ ). O processamento das amostras se deu por método automatizado $\left(\right.$ BACTEC $^{\circledR}$ modelos FX, série 9.000) que detecta o crescimento de microrganismos nas amostras de sangue. A identificação dos microrganismos e o perfil de sensibilidade antimicrobiana foram obtidos por cultura automatizada MicroScan ${ }^{\circledR}$ (Siemens). Foram consideradas hemoculturas por microrganismo resistente (HMC-MR): Staphylococcus aureus resistente à oxacilina (Methicillin-resistant Staphylococcus aureus - MRSA), Enterococcus spp resistente à vancomicina (Vancomycin-resistant enterococci - VRE), bactérias Gram-negativas resistentes a pelo menos uma classe dos antimicrobianos carbapenêmicos, cefalosporinas, fluorquinolonas e aminoglicosídeos. Hemoculturas cujos microrganismos eram sensíveis aos antimicrobianos foram denominadas HMC-MS.

Os dados de custo direto foram referentes ao consumo geral pelo paciente, ao longo da hospitalização e subdivididos nas categorias: medicamentos (antimicrobianos e outros), materiais usados na assistência do paciente e exames (laboratoriais e de imagens). O custo direto integral representou 0 somatória dos gastos com medicações, materiais e exames. $O$ custo direto total foi referente a todo período de hospitalização, enquanto o custo direto diário foi calculado dividindo-se o custo total da internação do paciente pelo seu respectivo número de dias de hospitalização6.

Os valores foram apresentados em reais (BRL - R\$) e dólares (USD - US\$). Para os valores em dólares foi considerado a cotação do mês de julho de 2017 (período mediano do estudo) no valor de $\mathrm{R} \$ 3,1301$.

Os dados desta pesquisa foram obtidos do prontuário eletrônico pelo programa Business Intelligence ${ }^{\circledR}$, responsável pela coleta e organização destes dados, que após processamento foram exportados para o programa Microsoft Exce ${ }^{\circledR}$, onde planilhas foram geradas e posteriormente analisadas pelo programa estatístico IBM SPSS Statistics versão 20.

Os resultados das variáveis contínuas foram descritos como mediana, média, desvio padrão (DP), dependendo da distribuição dos dados. O teste $U$ de Mann-Whitney foi utilizado para comparação das médias. As variáveis categóricas foram comparadas com o teste de Exato de Fisher. Para todos os testes o valor de $p<0,05$ foi considerado significativo, dentro do intervalo de confiança (IC) de 95\%.

Esta pesquisa está vinculada ao projeto "Impacto clínico e econômico da resistência antimicrobiana nos custos hospitalares", aprovado pelo Comitê de Ética em Pesquisa envolvendo Seres Humanos da Universidade Estadual de Londrina (Parecer $n^{\circ}$ 3.097.075) e Certificado de Apresentação para Apreciação Ética (nº 97120618.3.3001.5231).

\section{RESULTADOS}

A amostra do estudo foi composta por 261 pacientes com hemoculturas positivas e, destes, $62,45 \%$ apresentaram microrganismos resistentes aos antimicrobianos em pelo menos uma hemocultura (HMC-MR).

Os resultados demonstraram predomínio de idosos, hospitalizações pelo SUS, internações por motivos clínicos e permanên- 
Tabela 1. Associação das variáveis clínico-demográficas dos pacientes de acordo com o perfil de sensibilidade das hemoculturas

\begin{tabular}{|c|c|c|c|c|c|c|c|}
\hline \multirow[t]{2}{*}{ Variáveis } & Total (261) & $\begin{array}{c}\text { HMC-MR } \\
163(62,45)\end{array}$ & $\begin{array}{c}\text { HMC-MS } \\
98(37,55)\end{array}$ & \multirow[t]{2}{*}{ OR } & \multirow{2}{*}{\multicolumn{2}{|c|}{ IC $95 \%$}} & \multirow[t]{2}{*}{ p-valor* } \\
\hline & $n(\%)$ & \multicolumn{2}{|c|}{ Paciente (261) } & & & & \\
\hline \multicolumn{8}{|l|}{ Idade } \\
\hline$\geq 14$ anos $<65$ anos & $115(44,06)$ & $66(57,39)$ & $49(42,61)$ & & & & \\
\hline$\geq 65$ anos & $146(55,94)$ & $97(66,44)$ & $49(33,56)$ & 1,4697 & 0,8873 & 2,4343 & 0,0854 \\
\hline \multicolumn{8}{|l|}{ Tipo de convênio } \\
\hline Convênios/Particular & $37(14,18)$ & $18(48,65)$ & $19(51,35)$ & & & & \\
\hline SUS & $224(85,82)$ & $145(64,73)$ & $79(35,27)$ & 1,9374 & 0,9616 & 3,9035 & 0,0470 \\
\hline \multicolumn{8}{|l|}{ Tipo de internação } \\
\hline Cirúrgico & $101(38,70)$ & $62(61,39)$ & $39(38,61)$ & & & & \\
\hline Clínico & $160(61,30)$ & $101(63,13)$ & $59(36,88)$ & 1,0768 & 0,6444 & 1,7994 & 0,4389 \\
\hline \multicolumn{8}{|l|}{$\begin{array}{l}\text { Período de } \\
\text { hospitalização }\end{array}$} \\
\hline até 15 dias & $119(45,59)$ & $71(59,66)$ & $48(40,34)$ & & & & \\
\hline$>15$ dias & $142(54,41)$ & $92(64,79)$ & $50(35,21)$ & 1,2439 & 0,7524 & 2,0565 & 0,2346 \\
\hline \multicolumn{8}{|l|}{ UTI } \\
\hline Não & $70(26,82)$ & $33(47,14)$ & $37(52,86)$ & & & & \\
\hline Sim & $191(73,18)$ & $130(68,06)$ & $61(31,94)$ & 2,3895 & 1,3659 & 4,1801 & 0,0017 \\
\hline \multicolumn{8}{|l|}{ Permanência UTI } \\
\hline até 15 dias & $115(60,21)$ & $68(59,13)$ & $47(40,87)$ & & & & \\
\hline$>15$ dias & $76(39,79)$ & $62(81,58)$ & $14(18,42)$ & 3,0609 & 1,5370 & 6,0959 & $<0,001$ \\
\hline \multicolumn{8}{|l|}{ Desfecho clínico } \\
\hline Alta & $147(56,32)$ & $80(54,42)$ & $67(45,58)$ & & & & \\
\hline Óbito & $114(43,68)$ & $83(72,81)$ & $31(27,19)$ & 2,2423 & 1,3265 & 3,7904 & 0,0016 \\
\hline
\end{tabular}

Figura 1. Distribuição dos custos diretos totais em Reais atribuídos à hospitalização de pacientes de acordo com o perfil de sensibilidade das hemoculturas

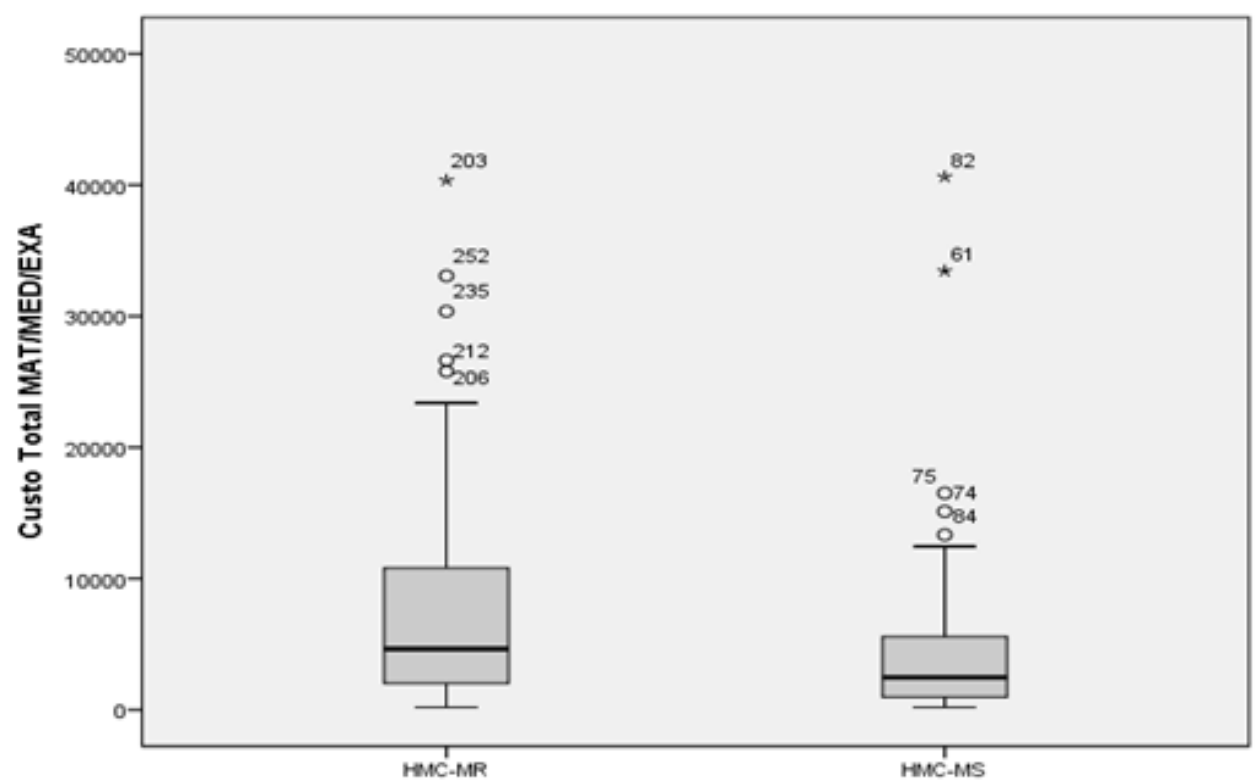

Legenda: HMC-MR: Hemocultura por microrganismo resistente; HMC-MS: Hemocultura por microrganismo sensível; MAT: materiais; MED: medicamentos; EXA: exames. $n=261$. 
cia em UTI por período superior a 15 dias. A resistência antimicrobiana aumentou em duas vezes as chances de mortalidade quando comparada aos pacientes com HMC-MS (Tabela 1).

Em relação ao tempo de hospitalização, não ocorreu diferença na média deste período entre os pacientes com HMC-MR e HMC-MS $(p=0,0629)$. A permanência em UTI, a média de dias de hospitalização nesse setor para os pacientes com HMC-MR foi de 15,86 dias enquanto para os pacientes com HMC-MS foi de 8,48 dias (OR 3,0609; IC 1,5370-6,0959; $p<0,001)$. A infecção resistente esteve associada a aumento em 7,38 dias na média de permanência em UTI $(p<0,001)$.

No que diz respeito ao custo total e diário, ambos foram superiores entre os pacientes com HMC-MR (Tabela 2).

Os pacientes com HMC-MR apresentaram maior custo direto (total e diário) na hospitalização $(p<0,001)$, quando comparados a pacientes com HMC-MS. A soma do custo direto total para todos os pacientes com HMC-MR foi de R\$1.214.491,35 e para os pacientes com HMC-MS foi de $\mathrm{R} \$ 432.461,31$. Apesar da diferença estatística foi possível observar a variabilidade na distribuição de valores gastos na internação dos pacientes, bem como a ocorrência de valores extremos em ambos os grupos (Figura 1).

Foram isolados 997 microrganismos das hemoculturas coletadas dos pacientes do estudo, sendo $679(68,10 \%)$ microrganismos sensíveis aos antimicrobianos e 318 (31,90\%) microrganismos resistentes, considerando que alguns pacientes apresentaram mais de um resultado positivo em hemocultura. (Tabela $3)$.
Quanto ao perfil de resistência antimicrobiana 57,55\% (183) eram resistentes aos carbapenêmicos, 34,90\% (111) possuíam resistência às cefalosporinas, fluorquinolonas e aminoglicosídeos, 5,03\% (16) eram Staphylococcus aureus resistente a oxacilina (MRSA) e 2,52\% (8) Enterococcus spp resistente à vancomicina (VRE).

\section{DISCUSSÃO}

Os resultados demonstraram que a resistência antimicrobiana esteve presente em mais da metade dos pacientes que apresentaram hemoculturas positivas para bactérias. Este dado se iguala a estudo brasileiro que analisou a prevalência e o perfil de sensibilidade antimicrobiana em hemoculturas de pacientes internados em UTI. Entre as hemoculturas positivas, $76,5 \%$ apresentavam bactérias multirresistentes ${ }^{7}$.

Entre os fatores que estiveram associados às HMC-MR, a hospitalização pelo SUS pode ser justificada pelo tipo de acomodação ofertada aos pacientes deste convênio, os quais ficam internados em enfermarias que possuem de 5 a 8 leitos na instituição onde o estudo foi realizado, sendo que as acomodações ofertadas aos pacientes de convênios e particulares variam de 1 a 2 leitos. O maior número de pacientes em enfermarias do SUS pode dificultar a prática de medidas de precaução de contato e isolamento, recomendadas aos pacientes infectados ou colonizados por MOMR $^{8}$.

Outro motivo pode ser justificado pelo perfil de gravidade de pacientes internados pelo SUS quando comparados aos hospita- 


\begin{tabular}{|c|c|c|c|c|c|c|}
\hline \multirow{5}{*}{ 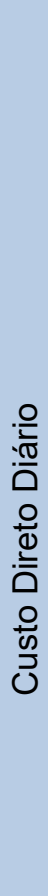 } & \multirow{3}{*}{$\sum_{\substack{0 \\
0}}^{\infty}$} & 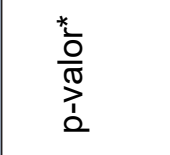 & $\begin{array}{l}\overline{8} \\
8 \\
0 \\
v\end{array}$ & 용 & $\begin{array}{l}0 \\
\varnothing \\
0\end{array}$ & $\begin{array}{l}5 \\
\overline{8} \\
0 \\
0\end{array}$ \\
\hline & & 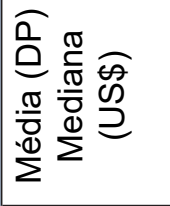 & 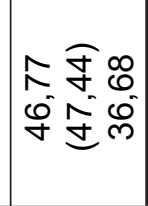 & 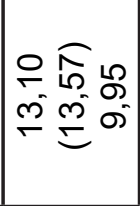 & 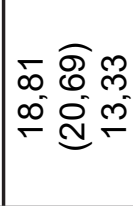 & 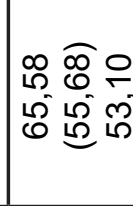 \\
\hline & & 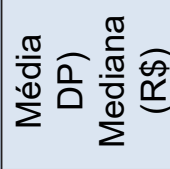 & 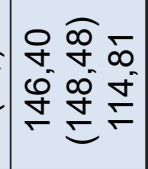 & 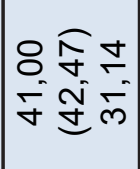 & 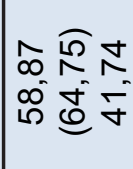 & 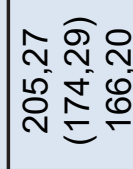 \\
\hline & $\sum_{i}^{\alpha}$ & 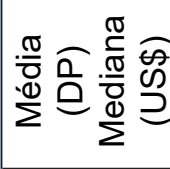 & 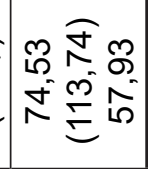 & 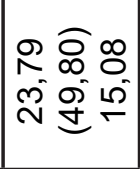 & 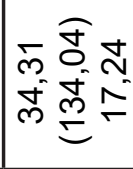 & 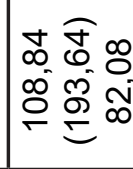 \\
\hline & $\sum_{\text {I }}$ & 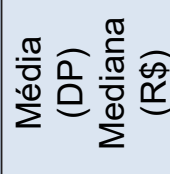 & 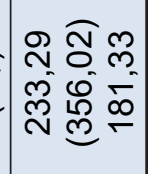 & 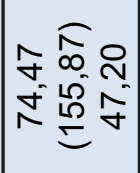 & 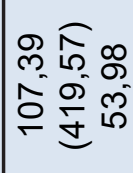 & 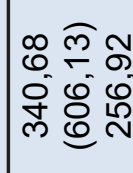 \\
\hline \multirow{6}{*}{ 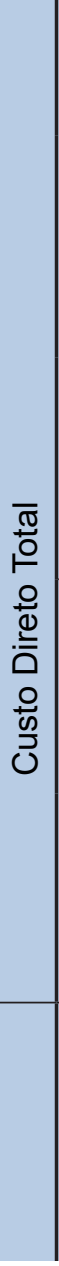 } & \multirow{3}{*}{$\sum_{\substack{0 \\
0}}^{\infty}$} & $\begin{array}{l}{ }^{*} \\
\frac{0}{\pi} \\
\stackrel{0}{10} \\
\stackrel{1}{\alpha}\end{array}$ & $\begin{array}{l}\overline{8} \\
0 \\
0\end{array}$ & $\begin{array}{l}\overline{8} \\
8 \\
0\end{array}$ & $\begin{array}{l}\overline{8} \\
8 \\
0 \\
v\end{array}$ & 㐫 \\
\hline & & 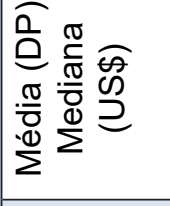 & 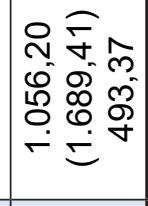 & 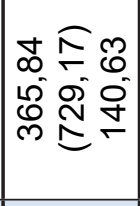 & 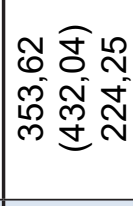 & 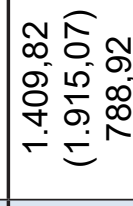 \\
\hline & & 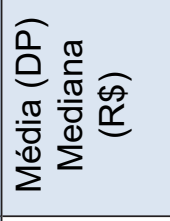 & 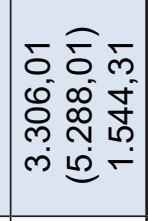 & 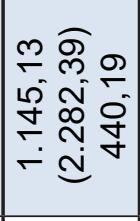 & 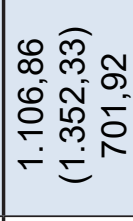 & 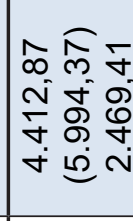 \\
\hline & $\sum_{0}^{\infty}$ & 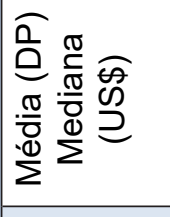 & 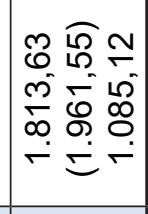 & 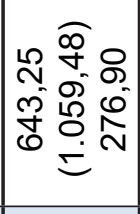 & 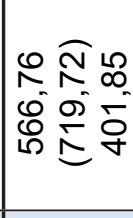 & 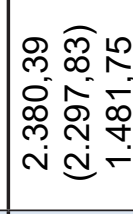 \\
\hline & $\sum^{\sum}$ & 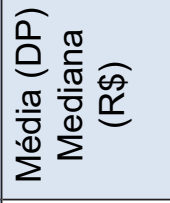 & 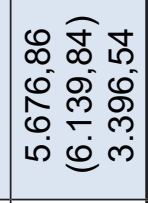 & 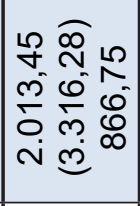 & 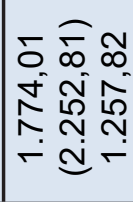 & 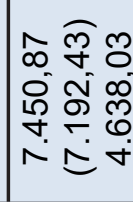 \\
\hline & & & 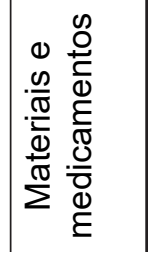 & 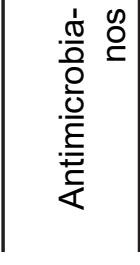 & \begin{tabular}{|l}
$\mathscr{\infty}$ \\
$\stackrel{\infty}{E}$ \\
$\stackrel{\mathbb{w}}{\widetilde{x}}$ \\
$\ddot{w}$
\end{tabular} & 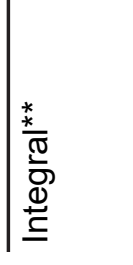 \\
\hline
\end{tabular}


Tabela 3. Distribuição dos microrganismos isolados nas hemoculturas de acordo com o perfil de sensibilidade dos antimicrobianos

\begin{tabular}{l|c|c}
\hline Microrganismos & $\begin{array}{c}\text { Total }=997 \\
\mathrm{n}(\%)\end{array}$ & $\begin{array}{c}\text { Resistentes aos antimi- } \\
\text { crobianos } \\
318(31,89 \%)\end{array}$ \\
\hline Serratia marcescens & $07(0,70)$ & - \\
\hline Streptococcus spp & $15(1,50)$ & - \\
\hline Providencia stuartti & $30(3,01)$ & $05(1,57)$ \\
\hline Proteus mirabilis & $35(3,51)$ & $03(0,94)$ \\
\hline Enterobacter spp & $64(6,42)$ & $29(9,12)$ \\
\hline Enterococcus spp & $76(7,62)$ & $8(2,52)$ \\
\hline Staphylococcus aureus & $80(8,02)$ & $16(5,03)$ \\
\hline Escherichia coli & $81(8,12)$ & $34(10,69)$ \\
\hline Pseudomonas aeruginosa & $113(11,33)$ & $23(7,23)$ \\
\hline Acinetobacter baumannii & $179(17,95)$ & $59(18,55)$ \\
\hline Klebsiella pneumoniae & $297(29,79)$ & $139(43,71)$ \\
\hline Outros & $20(2,01)$ & $02(0,63)$ \\
\hline
\end{tabular}

lizados por convênio ou particular. Estes últimos apresentam acesso direto aos serviços de saúde e usualmente procuram atendimento por quadro clínico de baixa complexidade, enquanto os pacientes assistidos pelo SUS, nessas mesmas condições, procuram as Unidades Básicas de Saúde ou as Unidades de Pronto Atendimento, para posteriormente serem encaminhados aos hospitais secundários e terciários diante do agravamento e complexidade do quadro.

Neste estudo, as HMC-MR não foram associadas à permanência hospitalar por período prolongado quando comparado aos pacientes com HMC-MS. Corroborando com estes achados, estudo europeu apresentou uma mediana de permanência hospitalar de nove dias para infecção por MOMR e de oito dias para microrganismo sensíveis aos antimicrobianos, mostrando menor impacto da resistência no aumento de dias de hospitalização ${ }^{9}$. A ausência na diferença do período de hospitalização também pode ser justificada considerando que no estudo atual todos os pacientes apresentavam HMC positivas, o que já indica um caso grave de infecção, independente do perfil de sensibilidade.

Outro fator associado à HMC-MR na presente pesquisa foi a hospitalização em UTI, com destaque para o período superior a 15 dias. $O$ aumento nos dias de permanência em UTI pode ser tanto causa quanto consequência da resistência antimicrobiana. Infecções desencadeadas por MOMR geram falhas terapêuticas que podem agravar $o$ quadro clínico do paciente, necessitando de cuidados intensivos. Por outro lado, longas internações em UTI demandam mais procedimentos invasivos devido à criticidade do paciente, consequentemente aumentam a 
exposição ao ambiente hospitalar e aos riscos de colonização/infecção por MOMR.

A hospitalização em UTI normalmente se destina a pacientes graves, em uso de antimicrobianos, o que favorece a pressão de seleção de cepas resistentes e consequentemente maior prevalência de MOMR neste ambiente. Corroborando este resultado, estudo mostrou que a resistência antimicrobiana foi mais frequente entre os pacientes internados em UTI e o tempo de permanência apresentou uma média de 12 dias $^{10}$.

A mortalidade apresentada neste estudo foi significativamente maior entre os pacientes com HMC-MR. Estudo epidemiológico realizado no mesmo munícipio da atual pesquisa mostrou que tanto a infecção como a colonização por MOMR foram associados à elevada mortalidade entre os pacientes hospitalizados, respectivamente $44,7 \%$ e $45,2 \%{ }^{11}$.

Na União Europeia, a resistência antimicrobiana é responsável por cerca de 25.000 mortes anualmente, apresentando custos adicionais nos cuidados de saúde e perda de produtividade no valor de pelo menos 1.500 milhões de euros ${ }^{4}$.

Em relação aos resultados de custos do atual estudo, foi considerado o consumo geral de medicações, os exames e materiais referentes às necessidades dos pacientes, ao longo da internação. Não foi possível identificar o consumo exclusivo para o tratamento da infecção multirresistente, limitação à análise de associação direta do custo com a resistência.

No presente estudo observou-se que os custos gerais diretos atribuídos à hospitalização foram maiores em pacientes com HMC-MR e, aproximadamente, duas vezes o valor da hospitalização de pacientes HMC-MS. Estes custos englobaram os medicamentos, exames e materiais utilizados no cuidado ao paciente. Todos eles foram superiores entre os pacientes com HMC-MR, mostrando que a resistência não impacta somente nos custos com antimicrobianos. Este resultado pode indicar que um paciente com infecção resistente realiza mais exames, tanto de imagens quanto laboratoriais, bem como recebem cuidados que requerem materiais mais custosos, em maior quantidade e por tempo prolongado. Neste estudo, o gasto excedente com os pacientes com HMC-MR foi de $\mathrm{R} \$ 782.030,04$ (US\$249.841,87) em comparação aos pacientes com HMC-MS.

Custos hospitalares excessivos também foram observados em estudo cujo custo total da resistência antimicrobiana atingiu $€ 109,3$ milhões e o valor médio por diária hospitalar entre os pacientes com infecções resistentes foi de $€ 1,103$ milhões $^{9}$.

Em relação aos custos hospitalares com antimicrobianos, estudo realizado entre pacientes com diagnóstico de infecção de corrente sanguínea por MRSA também mostrou que o custo total do tratamento antimicrobiano foi maior para os pacientes com infecção resistente (US\$1.061,01) do que quando comparados aos pacientes com infecção sensível (US $\$ 829,40)^{12}$.

Atualmente, a resistência antimicrobiana é um dos mais graves problemas de saúde pública e apresenta grandes consequências para a sociedade. O impacto dessa complicação infecciosa no ambiente hospitalar se traduz em elevada mortalidade e aumento dos custos diretos da hospitalização.

Os gastos com a saúde vêm crescendo 
em ritmo acelerado, podendo afetar inclusive a sustentabilidade dos sistemas de saúde, tanto privados como públicos. Nos últimos anos ocorreu um aumento expressivo das despesas do SUS com medicamentos, passando de $\mathrm{R} \$ 14,3$ bilhões em 2010 para quase $\mathrm{R} \$ 20$ bilhões em $2015^{13}$.

O tratamento com antimicrobianos, além de onerar os gastos públicos, é considerado uma grande preocupação em nível global quando utilizado de forma inadequada. Com a resistência antimicrobiana em ascensão, os tratamentos estão se tornando menos eficazes e as infecções persistentes e até mesmo incuráveis ${ }^{14}$.

Estes dados evidenciam a necessidade de investir em protocolos institucionais como o Antimicrobial Stewardship, para uso consciente dos antimicrobianos no tratamento das infecções hospitalares. Tal prática contribui para redução da seleção de microrganismos resistentes e custos, além de promover a saúde dos pacientes. Estudo mostrou que hospitais que adotam tais programas apresentaram redução de $85 \%$ nos gastos hospitalares e $92 \%$ nos custos com antimicrobianos, sendo

\section{REFERÊNCIAS}

1. Centers for Disease Control and Prevention (CDC). Antibiotic use in the United States, 2017: progress and opportunities [Internet]. Atlanta: US Department of Health and Human Services, CDC; 2017:1-40.

2. Interagency Coordination Group on Antimicrobial Resistance (IACG). Antimicrobial resistance: invest in innovation and research, and boost R\&D and access: IACG discussion paper [Internet]. Geneva: WHO; 2018:1 17. o principal fator de economia a queda no período de hospitalização ${ }^{15}$.

O conhecimento destas consequências por gestores e profissionais da área de saúde pode incentivá-los a adotar medidas custo efetivas para a prevenção da resistência antimicrobiana neste ambiente e consequentemente contribuir para melhorar a gestão dos recursos financeiros. Investir em ações educativas para o uso adequado de antimicrobianos, o controle e a prevenção de MOMR pode reduzir de forma significativa e sustentada a economia nos custos hospitalares e melhorar a qualidade da assistência à saúde.

\section{CONCLUSÃO}

A resistência antimicrobiana, detectada nos agentes etiológicos de hemoculturas, foi associada aos aspectos clínicos como a hospitalização prolongada em unidade de terapia intensiva e um aumento da mortalidade, bem como nos aspectos econômicos, cujos custos diretos da hospitalização foram maiores em pacientes com HMC-MR e, aproximadamente, duas vezes o valor da hospitalização de pacientes HMC-MS.
3. Costa ALP, Silva Junior ACS. Resistência bacteriana aos antibióticos e saúde Pública: uma breve revisão de literatura. Estação Cient (UNIFAP).

4. World Health Organization. Global action plan on antimicrobial resistance [Internet]. Geneva: World Health Organization; 2015:1-28

5. Agência Nacional de Vigilância Sanitária (ANVISA). Critérios diagnósticos de infecções relacionadas à assistência à saúde. Caderno 2 [Internet]. Brasília: ANVISA; 2017:1-86. 
6. Paula S, Corrêa V, Silva A. A contabilidade de custos e seu destaque na gestão. Rev Eletrônica Organ Soc [Internet]. 2019 [acessado em 10 out. 2019];8(9):125-39.

7. Fernandes DD, Oliveira MV, Paixão RS, Souza CL. Hemocultura em Unidade de Terapia Intensiva: prevalência, perfil de solicitações e susceptibilidade microbiana em um hospital do Sudoeste da Bahia. Rev Bras Pesq Saúde [Internet]. 2018 [acessado em 10 out. 2019];20(3):85-94.

8. Centers for Disease Control and Prevention (CDC). 2007 Guideline for isolation precautions: preventing transmission of infectious agents in healthcare settings [Internet]. Atlanta: CDC; 2019 [acessado em 10 nov. 2019].

9. Touat M, Opatowski M, Breun-Buisson C, Coster K, Guillemot C, Salomon J, et al. A payer perspective of the hospital inpatient additional care costs of antimicrobial resistance in France: a matched case-control study. Appl Health Econ Health Policy [Internet]. 2019.

10. Brixner B, Bierhals, ND, Oliveira CF, Renner JDP. Infecções da corrente sanguínea em unidade de terapia intensiva: estudo retrospectivo em um hospital de ensino. Enferm Atual Inderme [Internet]. 2019 [acessado em 10 out. 2019];87(25 Supl):1-7.

11. Souza ES, Belei RA, Maio Carrilho, CMD, Matsuo T, Yamada-Ogatta SF; Andrade, G, et al. Mortalidade e riscos associados a infecção relacionada à assistência à saúde. Texto Contexto Enferm [Internet]. 2015 [acessado em 05 nov. 2019];24(1):220-8.
12. Oliveira AC, Paula AO, Rocha RF. Custos com antimicrobianos no tratamento de pacientes com infecção. Av Enferm [Internet]. 2015 [acessado em 10 out 2019];33(3):352-61.

13. Vieira FS. Evolução do gasto com medicamentos do sistema único de saúde no período de 2010 a 2016. Texto para discussão: 2356 [Internet]. Brasília: IPEA; 2018 [acessado em 05 nov. 2019].

14. British Society for Antimicrobial Chemotherapy. Antimicrobial stewardship: from principles to practice. Birmingham, UK: BSAC; 2018.

15. Nathwani D, Varghese D, Stephens J, Ansari W, Martin $\mathrm{S}$, Charbonneau C. Value of hospital antimicrobial stewardship programs [ASPs]: a systematic review. Antimicrob Resist Infect Control [Internet]. 2019.

\section{CORRESPONDÊNCIA}

Gilselena Kerbauy

Av. Robert Koch, 60 - Londrina (PR), Brasil.

CEP: 86039-440

E-mail: gilselena@hotmail.com 